\title{
SOME THOUGHTS ON THE SAFETY ISSUES RELATING TO CHINESE HERBAL PRODUCTS
}

\author{
Dan Bensky and ERIGH Stöger
}

\begin{abstract}
As Chinese medicine becomes more and more integrated into the fabric of health care in economically developed countries, it runs into a variety of problems. These relate to education, licensing, and communications. In this article, we would like to focus on one important aspect - what does it mean to prescribe Chinese herbal medicines (CHM) responsibly at the present time?' This issue confronts all Chinese herbalists $(\mathrm{CH})$ in almost every patient encounter, and involves two major points that medical practitioners of all persuasions have to be cognizant of and which could be approached in a number of ways: safety and efficacy. How do we prescribe in a way that promotes the health of the patient without doing harm? We need to remember that all medicines, herbal or otherwise, have potential side effects and risks to patients, and that we need to take appropriate measures.
\end{abstract}

\section{Herb identification}

The first and most important issue that affects both safety and efficacy in CHM is herb identification. This is simply the knowledge of what precise plant or other substance is dispensed to the patient when a prescription is made. Given the plurality that is the core of CHM, it is not surprising that there are problems here. First and foremost among these is geography. Over the long history of CHM similar names were given to different plants in different parts of China. At the same time there are many cases of the same plant having multiple names. One example is the name 草河车 caoheche, which has been applied to at least two similar but functionally distinct herbs, Paridis Rhizoma (chonglou) and Bistortae Rhizoma (quanshen). This is one instance when standardised knowledge could be helpful. As has been born out in the infamous Belgian Slimming Cocktail Nephropathy

1 We have discussed these issues with a slightly different perspective in Bensky, Clavey, Stöger (eds) 2004, pp. xxiv-xxvii. 
incident discussed below, herb identification is vital for safety. It is of great importance also in practice. It is difficult to learn from experience in utilising an herb, if at different times different substances are dispensed as if they were the same.

\section{Herb quality}

While it is a truism that one cannot get quality results without quality herbs, evaluation of herbal quality is a very complicated and sophisticated endeavour that is far beyond the capabilities of anyone who is not trained in it. It involves more than the selection of the correct species of plant. It also pertains to such things as obtaining herbs from the right part of the country, harvested at the appropriate times, and processing correctly so that the herbs will have the maximum desired effect. For most practitioners the only real way to ensure quality of herbs is to use a reputable wholesaler or use prepared medicines from respected companies that at least use GMP (good manufacturing practice) procedures.

\section{Other basic safety issues in Chinese herbal medicine}

Herbs used in Chinese medicine are drawn from a very wide spectrum of substances that runs from food on one end to medicines that have a fairly tight therapeutic profile and require great forethought in prescribing (such as the various forms of aconite), on the other. For the purposes of this article, we will briefly cover the most common problems that may be encountered by patients taking Chinese herbal medicines. These can be divided into just three main categories. ${ }^{2}$

- Side effects: these are simple effects of the herbs that can be undesirable in a patient, even when given appropriately. For example, many herbs are oily (such as Angelicae sinensis Radix (danggui)) and lead to soft stools or diarrhoea in some patients.

- Toxic side effects: these are toxic effects that damage body tissues or organs. Almost always this occurs when the dosage is much greater

\footnotetext{
2 These general categories are dealt with in more detail in Ou Ming and Wang
} Ningshen 2002, pp. 2-17. 
than normal and when the duration of treatment with the herb in question is longer than normal. An example of this is Aconiti Radix lateralis preparata (zhifuzi), which is safe when prepared properly and given in an appropriate dosage, but can be deadly if not prepared correctly or with overdosages.

- Allergic reactions: these are infrequent and hard to predict interactions between specific herbs and specific people, mediated by the immune system. Since 1990 in China there have been almost 3,000 recorded incidents involving approximately 210 herbs. ${ }^{3}$ While this makes allergic reactions appear to be very rare, they do occur and practitioners need to always be on the lookout for them.

There are other important categories, such as safety during pregnancy, carcinogenic potential, etc. Many of the more serious side effects occur when the herbs are administered in ways not generally done in the west (such as intra-muscular or intravenous injections). However, in some cases large dosages of herbs can have detrimental effects, too. For example, there have been scattered reports of insomnia in patients taking $30 \mathrm{~g}$ per day of prepared Astragali Radix (zhihuangqi) that resolved when the herb was removed from the prescriptions. ${ }^{4}$ All of these require practitioners of CHM to keep current with the literature in this field, as well as pay close attention to their patients. As in all aspects of Chinese medicine, we must 'walk on two legs', giving respect both to the traditional literature and to modern biomedical research.

\section{Example: the case of Fangii}

This herb has been the focus of much interest since its implication in the Belgian slimming cocktail nephropathy (BSCN) tragedy of the early 1990's. The situation regarding the sources for this product is rather complex, as there are many different plants used under the name of 防己 fangï on the Chinese markets. Stephaniae tetrandrae Radix ( fangii) is the product listed in the Chinese Pharmacopoeia and is considered the standard. ${ }^{5}$ Aristolochiae fangchi Radix (guangfangii) has been used in China until recently, but it is being phased out as it

${ }^{3}$ Ou Ming and Wang Ningshen, 2002, p. 2.

${ }^{4}$ Chen and Chen 1997, pp. 44-5.

${ }^{5}$ State Pharmacopoeia Commission 2005, p. 101. 
has been in the West because it contains the known nephrotoxin, artistolochic acid. While the toxicity of that substance is probably significant only in large doses taken over an extended period of time, it should not be used until the relationship between its clinical and toxic effects are better understood.

In the case that brought the medical world's attention to this herb a group of Belgian physicians (who, as far as we know, had no real training in Chinese herbal medicine) combined a very large dosage (100-200mg) of what they thought was Stephaniae tetrandrae Radix ( fangii) with cascara powder $(20-150 \mathrm{mg})$, acetazolamide $(25-45 \mathrm{mg})$, Belladonna extract (1-2mg), and Magnoliae officinalis Cortex (100$200 \mathrm{mg}$ ), together with intradermal injections of artichoke extract and euphyllin and a low calorie diet. This large dosage of fangii, which was almost certainly Aristolochiae fangchi Radix (guangfangii) rather than the standard product Stephaniae tetrandrae Radix (fangi), demonstrates clearly a lack of knowledge of the basic attributes of the herbs in question. The end result was progressive renal fibrosis in many young women. ${ }^{6}$ While the entire approach was reckless, it is clear that the kidney damage only occurred after the Chinese herbs were added to the slimming cocktail, which demonstrates their contribution to the toxic mechanism. A recent study showed a high percentage $(46 \%)$ of urothelial carcinoma among 39 patients who had taken the Belgian slimming cocktails, which gives credence to the notion that large cumulative doses of herbs from the genus Aristolochia are a significant risk factor for urothelial carcinoma. ${ }^{7}$

All this shows that substances containing aristolochic acid can be dangerous and the removal from the pharmacopoeia of herbs that contain substantive amounts of it could be recommended. ${ }^{8}$ The reaction from governmental agencies was however predictably overreaching. Not only were any herbs whose names were remotely related to those containing aristolochic acid at least temporarily banned, but so were common and extremely useful herbs that may contain traces (but usually undetectable) of that substance. One

${ }^{6}$ While the draft by the Committee on Herbal Medicinal Products (2005, p. 6) states that over 100 cases of nephropathy were reported in young women from this slimming clinic, it gives no source for the data.

7 Nortier et al. 2000, pp. 1686-92.

${ }^{8}$ Note that the 2005 Chinese Pharmacopoeia still contains two herbs of the Aristolochiaceae family: Aristolochiae Fructus (madouling) and Aristolochiae Herba (tianxianteng). 
example is 细辛 xixin. It is true that the aerial parts of this plant have been found to have extremely low, but detectable, levels of aristolochic acid. ${ }^{9}$ It has therefore been considered prudent that only the terrestrial parts be used; it is no longer known as Asari Herba, but Asari Radix et Rhizoma in the new edition of the Chinese Pharmacopoeia. ${ }^{10}$ In our view this is an overreaction, as this herb is almost never prescribed in doses above $6 \mathrm{~g} /$ day and very rarely for any length of time.

While the toxicity of guangfangi is extreme, the response of regulatory agencies has been just as intense for other, much more benign or even nonexistent problems. The vast majority of the well-publicised problems with CHM have occurred as a consequence of gross over-dosage and 'off label' usage. Using any type of fangï for weight loss or ephedra-based supplements for increased energy have nothing to do with their usage in traditional forms of medicine. This does not seem to be understood by the regulators, and the resulting trickle-down hysteria has profound consequences for patients who could benefit from it. If a patient develops a problem subsequent to being treated by CHM, the immediate suspicion is now often that the Chinese herbs are the cause. This is true even when patients have also taken Western pharmaceuticals that could easily cause exactly the same problem. Part of the issue is that the Food and Drug Administration in the United States and the Medicines and Healthcare Products Regulatory Agency in the United Kingdom, the regulatory bodies controlling pharmaceuticals for hypertension, diabetes, and so on, trust western products by default. From what we have seen to date, however, the government functionaries who deal with CHM have a different opinion of our medicine.

\section{Interactions of Chinese herbal medicine with biomedicine}

A hotly contested topic at present is the issue of herb-drug interactions. This subject has only recently been given the attention it deserves, and in many cases the resulting guidelines remain unclear. In a recent book we pointed out that current discussion approaches the subject from the wrong end, similarly to discussing how pedestrians

${ }^{9}$ Cai Shao-Qing 2005, pp. 216-17.

${ }^{10}$ State Pharmacopoeia Commission 2005, p. 159. 
get in the way of automobiles. " For example, should grapefruit be outlawed due to its high potential of inducing side effects when ingested by patients taking a number of different drugs? Perhaps it would be more useful to approach the topic in a manner that gives the right of way to the pedestrians of the medical world, that is, herbal medicines.

There is another, perhaps even more insidious, form of adverse interactions involving Chinese medicine in interaction with conventional medicine. This is when ignorance of the efficacy of Chinese medicine by conventional medical practitioners can cause patients harm. Any Chinese herbalist who has used herbs as an adjunct to conventional cancer therapies will have many stories of patients who have been subjected to at least mild derision from oncologists with no understanding of the place of herbal treatments in this process. This is unhelpful and sometimes patients are given totally unnecessary medications simply because the oncologist cannot believe how well the patient is doing with herbal adjunctive treatment. In a similar vein, sometimes when patients with problems such as hypertension or Ménière's disease get benefit from CHM in a way that their biomedical practitioners find implausible, the patients may be prescribed unnecessary pharmaceuticals.

We will give another, more serious, example from our own experience. We once treated a patient whose cardiovascular disease was controlled very well for over ten years with Chinese medicine. He was involved in an accident that required orthopaedic surgery. Even though he told his doctors numerous times that he had cardiovascular disease and wanted either to continue his herbs or take alternative conventional treatment while in the hospital, they interpreted his history as meaning that he had no real cardiac problems. He underwent surgery without any complications and then died the next morning of a massive heart attack.

We must ensure that the standards applied to toxicities of Chinese herbal medicine are strict. However, it seems that when western pharmaceuticals lead to side effects, they are not subjected to the kind of treatment meted out to CHM. Certainly in the United States, pharmaceutical drugs take an enormous toll regardless of where they are dispensed. One recent study from a highly-computerised Veterans

"Bensky, Clavey, Stöger (eds) 2004, p. xxv. 
Administration Hospital noted that adverse drug events occurred in one quarter of all hospitalisations studied, and of these nine percent resulted in serious harm. ${ }^{12} \mathrm{~A}$ similar situation prevailed in an outpatient population, as shown by another recent survey, which found that amongst four groups of patients from different primary care practices in the Boston area, 25 percent had some adverse drug effects, 13 percent of which were serious. ${ }^{13}$

\section{Conclusion}

Chinese herbal medicines, like all forms of medicine, have potential side effects. These can vary from the trivial to the toxic, and all practitioners of CHM need to pay attention to these issues. There are patients who have the mistaken belief that if a treatment is natural, it must be completely safe. As trained professionals, we need to educate them on this issue and make sure that they are comfortable informing us of any adverse effects that occur. At the same time, we must also educate our colleagues who practise other forms of medicine about the relative safety of CHM in such a way that assures patients in the west have continued access to this effective form of health care.

\section{Note}

The authors would like to thank one of the anonymous referees for cogent and helpful criticisms of the draft.

\section{References}

Bensky, Dan, Steven Clavey, and Erich Stöger (eds) 2004, Chinese Herbal Medicine: Materia Medica, 3rd edition, Seattle: Eastland Press.

Cai Shao-Qing, Wang Xuan, Zhang Cui-Ying 2005, 'Comprehensive Studies on the Plants Containing Artistolochic Acids', in International Symposium in Beijing on Pharmacognosy, Beijing: School of Pharmaceutical Sciences, Peking University, 215-17.

${ }^{12}$ Nebeker et al. 2005, pp. 1111-16.
${ }^{13}$ Gandhi et al. 2003, pp. 1556-64. 
Chen Yingyi, Chen Chengli 1997, 'Two Cases of Insomnia Following Administration of Astragali Radix (服用黄花引起失㽢二例 Fuyong huangqi yinqi shimian erli.', Journal of the Anhui College of Chinese Medicine (安微中学院学报 Anhui zhongyixueyuan xuebao), 16, 3: 44-5.

Committee on Herbal Medicinal Products 2005, Public Statement on the Risks Associated with the use of Herbal Products Containing Aristolochia Species, London: European Medicines Agency.

Nortier, J.L. et al. 2000, 'Urothelial carcinoma associated with the use of a Chinese herb (Aristolochia fangchi)', The New England Journal of Medicine, 342, 23: 1686-92.

Ou Ming, Wang Ningsheng 2002, A Large Collection of Adverse Reactions from Chinese Medicinal Herbs and Preparations (中约及其制制不良反应大典 Zhongyao ji qi zhiji buliang fanying dadian), Shenyang: Liaoning Science and Technology Press.

Gandhi, T.K. et al. 2003, 'Adverse Drug Events in Ambulatory Care', New England Fournal of Medicine, 348, 16: 15-1564.

Nebeker J.R. et al. 2005, 'High rates of adverse drug events in a highly computerized hospital', Archives of Internal Medicine, 165, 10: 1111-16.

State Pharmacopoeia Commission 2005, Chinese Pharmacopoeia (中華人民共和國蓝典 Zhönghuá rénmin gönghéguó yàodiân), Beijing: Chemical Industry Press.

Zhu Jianhua 1991, Interactions between Chinese and Western Medicines (中西约物相互作用 Zhongxi yaowu xianghu zuoyong), Beijing: People's Health Publishing Company. 